\title{
Ultrashort ssDNA in Retinoblastoma Patients Blood Plasma Detected by a Novel High Resolution HPLC Technique: a Preliminary Report
}

\author{
Kirill V. Ermakov ${ }^{1, *}$, Alexander A. Bukhvostov ${ }^{1}$, Alexander S. Vedenkin², Sergey V. Stovbun², \\ Anton S. Dvornikov ${ }^{1}$, Dmitry A. Kuznetsov ${ }^{1,2}$
}

\section{ABSTRACT}

A significant population of ultrashort (50-150n) single-stranded DNA fragments were found in exosome-free blood plasma of retinoblastoma patients $\left(6.84 \mathrm{ng} \mathrm{mL}^{-1}\right)$, but not in plasma of healthy donors. An original high resolution HPLC technique has been proposed to reveal and characterize this peculiarity. To solve this task, a novel molecular size exclusion - anion exchange analytical technique was developed. Its applicability to diagnostics and oncogenesis research is quizzed here.

\section{KEYWORDS}

Retinoblastoma; blood plasma; cfDNA; ssDNA; HPLC

\section{AUTHOR AFFILIATIONS}

${ }^{1}$ N.I. Pirogov Russian National Research Medical University, Russian Federal Ministry of Health, Ostrovityanov St., 1, Moscow 117997 , Russia

2 N.N. Semenov Institute of Chemical Physics, Russian Academy of Sciences, Kosygin St., 4, Moscow 119991, Russia

* Corresponding author: N. I. Pirogov Russian National Research Medical University, Russian Federal Ministry of Health, Ostrovityanov St., 1, Moscow 117997, Russia; e-mail: ermakovkv07@gmail.com 


\section{INTRODUCTION}

The DNA repair machinery damage involves a marked DNA polymerase $\beta$ hyperexpression and its processivity limitations in retinoblastoma cells $(1,2)$. This may promote some tumor DNA release as long as the DNA repair related short polydeoxyribonucleotides are going to get lost in a chaotic modulation of chromatin structure (3-5).

So the DNA repair caused release of short single-stranded DNA sequences is likely to contribute to the blood circulated DNA pool in retinoblastoma patients.

To reveal such a contribution, the extra-high resolution ssDNA-detecting tool is needed. It is hardly possible to overestimate this requirement since the conventional agarose gel electrophoresis and PCR procedures are not selective and sensitive enough as long as the pg-ng concentration range for short (smaller than 100n) ssDNA detection in clinical samples is the case (1-5).

Taking this into account, the aim of a present work was to develop a new chromatographic technique suitable to solve the above specified task, a high resolution ssDNA analysis in liquid biopsies.

\section{MATERIALS AND METHODS}

Four 5.0 year old male retinoblastoma (2A) patients and four adult male healthy donors were taken for blood plasma cfDNA extraction according to $(4,5)$. A consequent treatment of DNA extract with exonucleases lambda, III and S1 (3) was followed then by a cascade ultrafiltration on K75/K25 SPM TechSep (Mirabel, France) membranes (6). An enzymatic treatment of samples was conventionally carried out to purify ssDNA (3) aiming for a double-stranded DNA hydrolysis followed by ultrafiltration removal of the resulted free nucleotides and nucleases 1, III traces $(3,6)$. As a negative control, the ssDNA destroying nuclease S1 treatment has been employed (3). The enzymatic treatment steps were performed to engage a known peculiarities of phage 15 '-exonuclease and E. coli 3'-exonuclease III, both digesting double-stranded DNA (7) and Aspergillus orizae nuclease S1 digesting ssDNA (7). All enzymes were purchased from Worthington Corp., USA. Enzymatic treatment regime: $20 \mathrm{mM}$ Tris- $\mathrm{HCl}$ (pH 8.40), $10 \mathrm{mM} \mathrm{MgCl}$, $20 \mathrm{mM} \mathrm{NaCl}, 10 \mathrm{mM}$ DTT, 15 mM EDTA, $60 \mathrm{~min},+37^{\circ} \mathrm{C}(3,6,7)$. A resulted fraction of lambda/ III-nuclease resistant $25-75 \mathrm{kDa}$ compounds was analysed by size exclusion/anion exchange (SEAE) HPLC.

For this purpose, our original procedure has been employed. Its key parameters are the followings: stationary phase - polymethylamidopropylmethacrylamide; column PRP-X600 AE, $4.6 \times 150.0 \mathrm{~mm}, 5.0$ micrometer particles, $1.6 \mathrm{meq} / \mathrm{mL}$ (Hamilton Corp., USA); 1,800 p.s.i., $22-25^{\circ} \mathrm{C}$, $0.8 \mathrm{~mL} / \mathrm{min}$ elution rate. Both synchronous linear elution $\mathrm{LiCl}_{2}(0-2.5 \mathrm{M})$ and $\mathrm{pH}$ (8.0-4.0) gradients were formed on $100 \mathrm{mM}$ Tris/acetonitrile (85:15, v/v). Waters/Hamilton compatible Breeze 200SLE Analytical System, W2998 UV-Detector $(254 \mathrm{~nm})$, W600E gradient former (Waters, Inc., USA). Sample loading: 80-100 g DNA in 50 microliters of $100 \mathrm{mM}$ Tris- $\mathrm{HCl}$ ( $\mathrm{pH} 8.0)$ /acetonitrile (85:15, v/v). All ssDNA measurements and $2.0 \%$ agarose gel electro- phoresis DNA size control were performed as described in (3).

For a positive control, the mixtures of equal amounts (5.0-6.0 ng/mL) of different size Poly (dT) single-stranded sequences were applied onto a column. Poly(dT)50, Poly(dT)100 and Poly(dT)150 species (ThermoFisher Corp., USA) were employed.

\section{RESULTS}

The content of ssDNA in the blood plasma of patients is $6.84 \pm 0.56 \mathrm{ng} \mathrm{mL}^{-1}$, this DNA population consists of ultrashort fragments (50-150n). In the control, a smaller population of ssDNA $\left(2.40-2.82 \mathrm{ng} \mathrm{mL}^{-1}\right)$ was found consisting of the larger 350-400n sequences (Figure 1). Besides, the blood circulating ssDNA population is found to be heterogeneous in retinoblastoma patients but not in healthy donors (Figure 1).

Noteworthy, the retention time (Rt) values detected in a positive control fractionation runs (see Methods) were found equal to 19-20 min (Poly(dT)50), 16-17 min (Poly (dT)100) and 12-13 min (Poly(dT)150).

\section{DISCUSSION}

A separation efficiency shown by our original SEAE-HPLC technique allows to reveal the size/charge - different populations within the ssDNA pool in a cancer patient blood plasma (Figure 1). Noteworthy, this fractionation procedure provides with a far better resolution level as com-

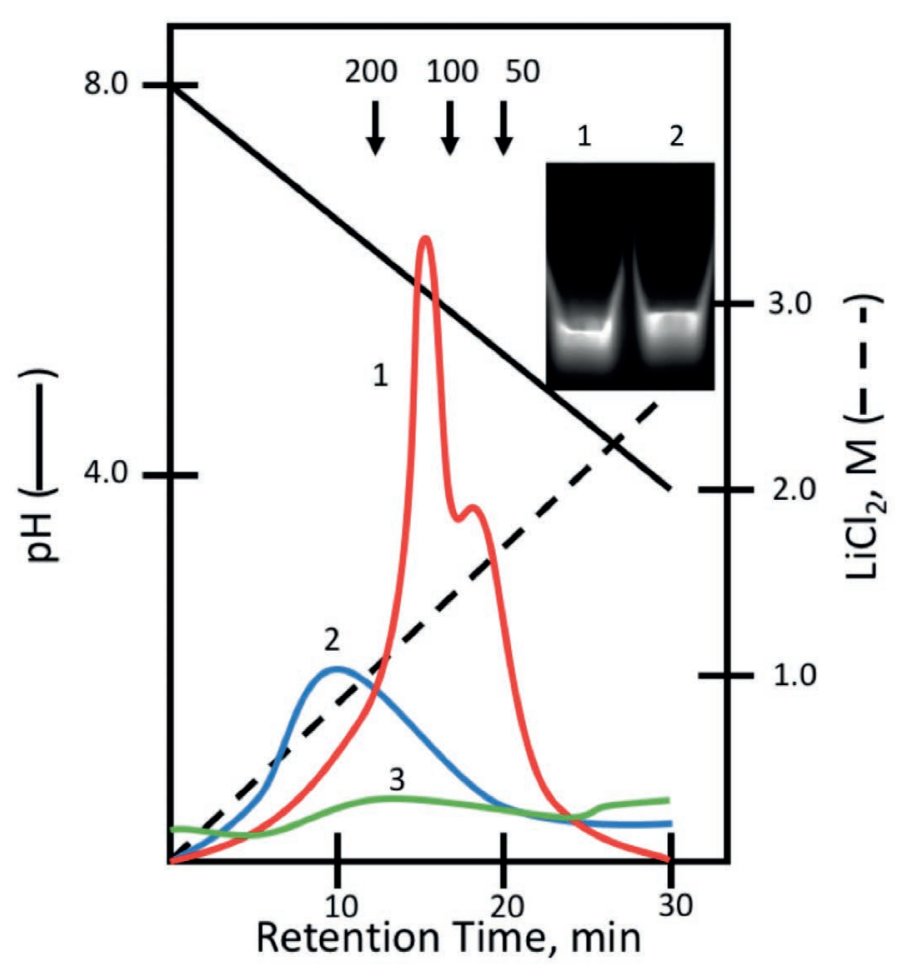

Fig. 1 Fractionation of plasma ssDNA fragments by HPLC on Polymethylamidopropylmethacrylamide. 1 - retinoblastoma patient, 2 - healthy donors, 3 - S1 nuclease control. Arrows show the retention times for 50n, 100n and 200n single-stranded poly (dT) markers (Takara Biomedical Corp., Rep. Korea). 
pared to the APCR engaging DNA size measurements and/ or to a routine agarose gel electrophoresis (3-5). Moreover, the PCR engaging DNA size measurement might be an inadequate choice once these DNA fragments have the DNA repair related origin. In this case, the release of ssDNA species would occur in the DNA repair process usually accelerated in malignancies (1-4).

So an original procedure of SEAE-HPLC we proposed can be used then in biochemistry as well as in molecular biology, biotechnology, experimental oncology, analytical and clinical chemistry. Paying attention to a clear heterogeneity of ssDNA pool within the 50-150n molecular size range (Figure 1), a certain advantage of our HPLC analytical tool should be emphasized.

As per the positive control findings (see Results), it seems nothing but a clear sign of high resolution capabilities of our technique.

The molecular lengths of smallest ssDNA ever found in the human blood plasma cfDNA pool, either healthy or any disease diagnosed patients (cancer, infections, autoimmune pathologies) were not smaller than $770 n$, contents level 6.5-18.0 $\mathrm{ng} \mathrm{mL}^{-1}(4,5,9)$. This is the reason why we prefer the term "ultrashort" to pay attention to uniqueness of two facts: (1) high resolution of HPLC technique we proposed and (2) very small sizes of ssDNA detected (50-150n). The latter fact, as well as a clear heterogeneity of the retinoblastoma patients ssDNA pool (Figure 1), is an attention catching point with no any reliable explanation beyond. Obviously, this should be a part of agenda of our forthcoming studies.

A probable diagnostic potential of ultrashort ssDNA as the early markers capable of appearing in the blood plasma prior to protein oncomarkers should be further investigated.

The question we might expect: Would it be a possible to claim that our novel method might specifically detect ultra-short cell-free DNA (cfDNA) from retinoblastoma patients? The answer is "no". Not just cfDNA of a certain size but, exclusively, their initially originated single-stranded species (ssDNA) are in a focus here with an emphasize on their possible DNA-repair related origin.

A remarkably high detection sensitivity of our SEAE-HPLC technique is no doubt helpful for further studies aiming to find out a link between the DNA repair damages in carcinogenesis and the appearance of the broken DNA repair products, short ssDNA $(1,2)$, in the patients blood plasma. So our method is to be treated as a reliable tool for both oncogenesis research and laboratory diagnostics purposes.

To the best of our knowledge, this is a first report ever on 50-150n ssDNA fragments presented in reliably detected ultra-low concentrations in cancer patient blood plasma to the contrast with healthy controls. On another hand, it should be safe to say that the present study is just a preliminary technical report with a certain potential for further clinical and experimental research.

It should be also outlined that this work deals with a new, previously not known (3-5) combination of molecular size exclusion and anion exchange separation principles in detection of a certain ssDNA populations within a clinical liquid biopsy samples. This itself is a key technological message of a present study.
Our assumption on the DNA repair related origin of the tumor-released ultrashort ssDNA fragments deserves further extensive studies being a new contribution to understanding of the carcinogenesis molecular mechanisms.

Comparing our method with a massive sequencing (MPS) technology (8), we have to state that a random, indiscriminate, nature of the DNA repair resulted short ssDNA fragments makes MPS limited to the DNA size estimation only. A primary structure of DNA chains is not in a focus once the DNA repair related origin of ssDNA has been assumed. So our HPLC approach might be treated as a reasonable alternative to MPS. Being about the same sensitive as MPS, our SEAE-HPLC technique provides nearly the same level of resolution available at $\sim 30-40 \%$ lower expenses compared to MPS, once an appropriate HPLC system is in a lab equipment list.

Being a preliminary result, this work is an attention catching signal for those ones involved into a long lasting cfDNA studies of all sorts (3-5) including clinical and analytical chemistry, molecular medicine, etc. After all, this is the first report ever on a SEAE-HPLC version "nicely applicable" for analytical purposes once the blood circulated short ssDNA fragments are in a focus.

\section{CONCLUSIONS}

A new chromatographic procedure described is to be taken as an efficient tool for short ssDNA sequences detection in biomaterials which itself means a promising approach to ongoing studies on diagnostic relevance of cfDNA species in oncology.

Noteworthy, a resolution capabilities of this HPLC procedure look better as compared to most known chromatographic DNA separation versions (3), PCR and agarose gel electrophoresis $(4,5)$.

This work presents data of unconventional cell-free ssDNA isolation that should be of interest to the liquid biopsy technology community.

\section{ABBREVIATIONS}

SPM - sulfonated polysulfone membranes

PRP - polymethylamidopropylmethacrylamide

cfDNA - cell-free DNA

ssDNA - single-stranded DNA

SEAE - size exclusion - anion exchange

\section{REFERENCES}

1. Bukhvostov AA, Dvornikov AS, Ermakov KV, Kurapov PB, Kuznetsov DA. Retinoblastoma: magnetic isotope effects might make a difference in the current anti-cancer research strategy. Acta Medica (Hradec Kralove) 2017; 60: 93-6.

2. Bukhvostov AA, Dvornikov AS, Ermakov KV, Kuznetsov DA. Retinoblastoma case: shall we get a paramagnetic trend in chemotherapy? Arch Cancer Res 2017; 5: 158-61.

3. Dewar JM, Lyndall D. Simple non-radioactive measurement of single-stranded DNA. In: Bjergbaek L, ed. Methods in molecular biology: DNA repair protocols. Totowa NJ: Humana Press, 2012; 920: 341-8.

4. Vong JSL, Tsang JCH, Jilang P, Lee WS, Leung TY, Chan KCA, Chiu RWK, Chiu YM, Lo D. Single-stranded DNA library preparation pref- 
erentially enriches short maternal DNA in maternal plasma. Clin Chem 2017; 63: 1031-7.

5. Fernando MR, Jiang C, Kryzanowski GD, Ryan WL. Analysis of human blood plasma cell-free DNA fragment size distribution using EvaGreen chemistry based droplet digital PCR. Clin Chim Acta 2018; 483: 39-47.

6. Aimar P, Meireles M. Calibration of ultrafiltration membranes against size exclution chtomatography columns. J Membr Sci 2010; 346: 233-9.
7. Dupret JS, Leroux AM. Nucleases. University of Montpellier: Montpellier, 2016.

8. Baglioni C, Andreotti G. Methods in DNA Research. Universita Cattolica del Sacro Cuore Publ.: Rome, 2017.

9. Levi IG, Yagel S, Gorowitz A. Extracellucar DNA. Abraham Weizman Institute of Science Publ.: Rehovoth, 2018. 